\title{
Map location affects center-surround modulation in a network model of VI
} Marcel Stimberg* and Klaus Obermayer

\author{
Address: School of Electrical Engineering and Computer Sciences, Technische Universität Berlin and Bernstein Center for Computational \\ Neuroscience, Berlin, Germany \\ Email: Marcel Stimberg* - mst@cs.tu-berlin.de \\ * Corresponding author
}

from Eighteenth Annual Computational Neuroscience Meeting: CNS*2009

Berlin, Germany. 18-23 July 2009

Published: 13 July 2009

BMC Neuroscience 2009, I0(SuppI I):PI79 doi:I0.II86/I47I-2202-I0-SI-PI79

This abstract is available from: http://www.biomedcentral.com//47I-2202/I0/SI/PI79

(C) 2009 Stimberg and Obermayer; licensee BioMed Central Ltd.

The response of a cell in primary visual cortex does not only depend on the stimulus presented in the classical receptive field (CRF), but is also modulated by the spatial context. The strength and sign of this modulation depends on the properties of the center and the surround stimulus, most notably their contrast and orientation. Our goal in this study is to gain further insight into the neuronal mechanisms shaping the response to such extra-classical stimulation.

Likely anatomical substrates for surround modulation are the lateral long-range connections and the feedback connections from higher visual areas [1]. However, it is also known that the local recurrent network within a single hypercolumn strongly shapes the response of a neuron. In a recent modeling study $[2,3]$ with a realistic orientation map layout, it was shown that only a network with strong recurrent connections is compatible with physiological data on orientation tuning. The previous study was restricted to the analysis of local computations, corresponding to stimulation of the CRF; here, we extend this study by determining the influence of the local network on modulatory surround effects. To this end, we extend the network by including an additional, orientationtuned surround input as an effective description of the surround influence.

We present an exploration of the parameters of this surround input, such as the strength of input to the excitatory respectively the inhibitory subpopulation and its tuning width. Depending on these parameters, various forms of suppression and facilitation can be observed. In particular, although the direct surround input is the same for all cells with a certain preferred orientation, the strength and tuning of modulation may change with map position. This is due to the local network connectivity: A cell's response is not only modulated directly by the surround input, but also indirectly by the change in response in neighboring cells. This neighborhood may be functionally very similar (in linear domains of the orientation map) or very diverse (close to singularities of the map). Therefore, a complete description of surround modulations cannot neglect the local cortical circuit. This finding is compatible with recent physiological findings in cat V1 showing that the surround influence is more broadly tuned in cells close to singularities of the orientation map.

\section{References}

I. Angelucci A, Bressloff PC: Contribution of feedforward, lateral and feedback connections to the classical receptive field center and extra-classical receptive field surround of primate VI neurons. Prog Brain Res 2006, 154:93-120.

2. Stimberg M, Wimmer K, Martin R, Schwabe L, Mariño J, Schummers J, Lyon DC, Sur M, Obermayer K: The operating regime of local computations in primary visual cortex. Cereb Cortex 2009 in press.

3. Wimmer K, Stimberg M, Martin R, Schwabe L, Mariño J, Schummers J, Lyon DC, Sur M, Obermayer K: Dependence of orientation tuning on recurrent excitation and inhibition in a network model of VI. In Advances in Neural Information Processing Systems 21 MIT Press; 2009 in press. 